\title{
EL POSTPOSITIVISMO DE LA «OPTIMIZACIÓN»: SOBRE EL CONCEPTO DE PRINCIPIO JURÍDICO DE R. ALEXY*
}

\author{
Alí Lozada \\ Universidad de Alicante
}

RESUMEN. El propósito del trabajo es proponer un esbozo reconstructivo de la teoría de los principios como mandatos de optimización, a fin de apuntar algunas observaciones críticas. La idea reconstructiva rectora es que la optimización de los principios no significa sino el uso ineludible de ciertos modelos argumentativos en la práctica jurídica, en los cuales los principios cumplen papeles operativos típicos, que les son definitorios. La caracterización de tales modelos, por un lado, permite descartar ciertas interpretaciones originadas en la equivocidad del término "optimización», y por otro, deja vislumbrar algunas insuficiencias en el postpositivismo de Robert ALEXY.

Palabras clave: ALEXY, optimización, ponderación, postpositivismo, principios, proporcionalidad, razones para la acción, valores.

\section{Postpositivism of «optimization»: on the concept of legal principle of R. Alexy}

ABSTRACT. In this paper we find a critical approach to the theory of principles as optimisation commands. For this purposes the author proposes a reconstructive draft revolving on the idea that the optimisation of principles implies the necessary use of certain kind of argumentative models in legal practice; models in which principles play their very typical operative role. Characterizing such models will allows us, on the one side, to reject certain interpretations based on the equivocal meaning of the term «optimisation» and, on the other, to become aware of some insufficiencies of Robert ALEXY's version of post-positivism.

Keywords: ALEXY, optimisation, balancing, post-positivism, principles, proportionality, reasons for action, values.

\footnotetext{
* Fecha de recepción: 18 de julio de 2016. Fecha de aceptación: 4 de agosto de 2016.
} 


\section{¿Qué hombre sabio, si se le forzara a escoger entre vivir sin rosas y vivir sin coles, no aseguraría las coles? George Bernard SHAw (The Apple Cart. A Political Extravaganza)}

\section{INTRODUCCIÓN}

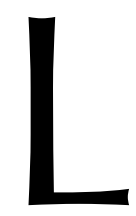

as últimas han sido décadas de emergencia y desarrollo del denominado postpositivismo ( $v$. gr., en ATIENZA y RUIZ MANERO, 2009: 259ss.), o constitucionalismo argumentativo (v. gr. en ATIENZA, 2007: 115ss.; y FERRAJOLI, 2011: 20). Esta concepción iusfilosófica asume al derecho como una práctica social dotada de una dimensión argumentativa que le es troncal, de manera que la tesis postpositivista fundamental sería la de que hay una vinculación argumentativa necesaria entre la práctica del derecho y la práctica de la moral (sobre esto, vid. LOZADA, 2016: 17 ss.); lo que supone que, en la práctica jurídico argumentativa, no solamente operan resortes autoritativos sino también morales. Para dar cuenta de esto, el postpositivismo ha acuñado una categoría que le es cardinal, la de principio jurídico: estos serían una suerte de bisagra (o de «puente», en términos de ALEXY) que articula la práctica justificativa del derecho con la de la moral. Por eso, la gran mayoría de los autores postpositivistas (pensemos en Alexy, Atienza, Dworkin, Ruiz Manero, etc.) han propuesto sus respectivas versiones teóricas de los principios jurídicos.

Una de las claves centrales - por tanto- para el estudio crítico del postpositivismo, y para su eventual desarrollo, está en el examen de las teorías de los principios defendidas por los diferentes autores de aquella corriente iusfilosófica, encaminado a elucidar cuál es el mínimo común denominador de todos ellos, es decir, el modelo principialista general, y cuáles son las peculiaridades de los diferentes modelos particulares. Pretendo, con este trabajo, dar un paso «inevitablemente» corto en esa dirección: me ocuparé del concepto de principio jurídico de ALEXY, limitándome a proponer un esbozo de reconstrucción de los principios como «mandatos de optimización» y a barruntar algunas observaciones críticas.

\section{GÉNESIS DE LA IDEA DE «MANDATOS DE OPTIMIZACIÓN»: LA CRÍTICA A DWORKIN COMO PUNTO DE PARTIDA}

Como es bien conocido, ALEXY publicó en 1978 su Teoría de la argumentación jurídica (Theorie der Juristischen Argumentation). No obstante, como el propio autor constata, «la ponderación no fue un tema dentro de [esa obra...]. El concepto de ponderación (Abwägung) no aparece siquiera en el índice temático. [...] El concepto de principio, a diferencia del de ponderación, sí [...]. Sin embargo, los principios se encuentran definidos de forma contraria a lo que la teoría de los principios [la del propio autor] sostiene, es decir, están definidos como "enunciados normativos con un alto nivel de generalidad"» (ALEXY, 2011a: 67). El desarrollo propiamente de la teoría alexyana de los principios arranca con su artículo de 1979, Sobre el concepto de principio jurídico (Zum Begriff des Rechtsprinzips), en el que aborda críticamente la dis- 
tinción entre reglas y principios trazada por DWORKIN en su célebre trabajo El modelo de las reglas (The Model of Rules), de 1967.

Recordemos que el meollo de la distinción dworkiniana radicaba en lo siguiente: las reglas son aplicables a la manera de todo-o-nada; mientras que los principios presentan la dimensión del peso o importancia (vid. DwORKIN, 1967). A este respecto, AlEXY observa, en primer lugar, que aquella «no es una distinción de grado. Los criterios de distinción no son comparativos, sino estrictamente clasificatorios. Eso significa», añade AlEXY, «que la frecuentemente problemática distinción de acuerdo con el grado de generalidad [...] es rechazada por DwORKIN», quien defiende una «tesis fuerte de la distinción»: «la distinción entre reglas y principios no es una distinción de grado, sino que reglas y principios son normas con estructuras lógicas completamente diversas» (ALEXY, 2014: 207). Sin embargo, en opinión de ALEXY, el señalado carácter todo-o-nada es inepto para distinguir en sentido fuerte las reglas de los principios, ya que depende de que todas las posibles excepciones a las reglas sean enumerables de antemano, lo que es imposible; como también lo es, en los ordenamientos jurídicos modernos, contar exclusivamente con reglas que no aceptan excepciones; cuando más, lo que puede hacerse es incorporar en ellas cláusulas generales de reserva como, por ejemplo «si no está exigido algo distinto a partir de otras razones jurídicas», lo que si bien dotaría de carácter todo-o-nada a las reglas, lo haría en un sentido que también lo tendrían los principios, ya que también en estos se pueden incorporar cláusulas como, por ejemplo «si ningún otro principio, con resultado contradictorio, tuviera precedencia» (ibid., 209 y ss.). ALEXY considera, en cambio, que la dimensión del peso atribuido por DWORKIN a los principios sí provee un criterio sobre el que erigir la tesis fuerte de la distinción que nos ocupa, ya que dicho peso se manifiesta en la manera en que los principios colisionan entre sí, marcando una diferencia cualitativa con la forma en que las reglas conflictúan unas con otras (ibid., 213 y ss.). En el artículo de 1979, además, ALEXY bosqueja las ideas básicas de su propia teoría de los principios: para él, la clave para conceptualizarlos, yendo más allá de DwORKIN, está en preguntarse por qué los principios cuando colisionan se comportan en la forma peculiar ya descrita por el autor norteamericano. Su respuesta embrionaria fue que eso tiene que ver con que «los principios mandan, prohíben o permiten algo que puede ser más o menos cumplido», lo que «se expresa claramente a través del principio de proporcionalidad. Característico de tales prescripciones es, por tanto,» escribe ALEXY, «el hecho de que ellas contienen mandatos de optimización». Y complementa: «En vez de mandatos de optimización, en alusión por ejemplo a la utilización de estos conceptos por MOORE, VON WRIGHT y SCHELER, se podría hablar de "deber ser ideal" o "ideales"», concepto que, al decir de AlEXY, «puede ser usado para esclarecer el carácter prima facie especial de los principios» (ibid., 221 y ss.). La «optimización» viene a ser, entonces, el meollo de la distinción alexyana entre reglas y principios.

Como bien se sabe, ALEXY desarrolla su teoría de los principios en su Teoría de los derechos fundamentales, publicada en 1985. Alli formula su tesis fuerte de la distinción entre principios y reglas. Los primeros serían «mandatos de optimización», en el sentido de que «son normas que ordenan que se realice algo en la mayor medida posible, en relación con las posibilidades jurídicas y fácticas [...Ellos] están caracterizados por el hecho de que pueden ser cumplidos en diferente grado y que la medida debida de su cumplimiento no solo depende de las posibilidades reales sino también de las jurí- 
dicas». Mientras que las reglas serían «mandatos definitivos», es decir, «normas que exigen un cumplimiento pleno y, en esa medida, pueden siempre ser solo o cumplidas o incumplidas. Si una regla es válida, entonces es obligatorio hacer precisamente lo que ordena, ni más ni menos. Las reglas contienen por ello determinaciones en el campo de lo posible fáctica y jurídicamente». De esta manera, nuestro autor traza una distinción cualitativa y no de grado (como la basada en el criterio de generalidad); en cuya virtud, «[t]oda norma es o bien una regla o un principio». (ALEXY, 2002b: 86 y ss.), proporcionando así, como afirma RUIZ MANERO, «una explicación de la distinción entre reglas y principios que no es incompatible con la de DwORKIN, sino de un grado superior a esta: la diferencia entre ambos tipos de estándares anotada por ALEXY da cuenta del porqué de las diferencias anotadas por DwORKIN» (RUIZ MANERO, 1990: 159).

Al decir de ALEXY, la distinción tal como él la propone se manifiesta en la diferencia estructural en que se solucionan, por un lado, los conflictos de reglas y, por otro, las colisiones de principios: «Un conflicto entre reglas», escribe ALEXY, «solo puede ser solucionado o bien introduciendo en una de las reglas una cláusula de excepción que elimina el conflicto o declarando inválida, por lo menos, una de las reglas» (ALEXY, 2002b: 88). «Cuando dos principios entran en colisión», en cambio, «uno de los dos tiene que ceder ante el otro. Pero esto no significa declarar inválido al principio desplazado ni que en el principio desplazado haya que introducir una cláusula de excepción. Más bien, lo que ocurre es que, bajo ciertas circunstancias, uno de los principios precede al otro. Bajo otras circunstancias, la cuestión de la precedencia puede ser solucionada de manera inversa» (ibid., 89). El examen de la teoría alexyana de los principios va a consistir, entonces, en la elucidación de qué debe entenderse por «mandatos de optimización» y de cómo este concepto configura los modelos argumentativos para la solución de colisiones de principios.

\section{3. «MANDATOS» «DE» «OPTIMIZACIÓN»Y «MÁXIMA DE LA PROPORCIONALIDAD»}

En Teoría de los derechos fundamentales, ALEXY explica que cuando él sostiene que los principios son «mandatos», está empleando este término «en un sentido amplio, que abarca también permisiones y prohibiciones» (ALEXY, 2002b: 86). Sin embargo, no realizó ninguna explicación similar acerca de qué quiere decir con que tales mandatos son «de» optimización. De esto se ocupó en Sobre la estructura de los principios jurídicos (Zur Struktur der Rechtsprinzipien), trabajo publicado en 2000. Allí contestó a la siguiente crítica de AARNIO: «Un mandato, de acuerdo con el concepto [de ALEXY], es como una regla: o se sigue o no se sigue. De este modo, el mandato de optimización es también una regla que no puede ser aplicada "más o menos". O se optimiza o no se optimiza. Por ejemplo, en caso de conflicto entre dos principios valorativos, los principios deben ser armonizados de manera óptima y solo de esta manera» (ALEXY, 2003d: 27). La respuesta de ALEXY fue que «[r] esulta necesario diferenciar los mandatos que se optimizan y los mandatos de optimización. [...] Los principios, en tanto objetos de la ponderación, no son entonces mandatos de optimización, sino mandatos que se optimizan o mandatos a optimizar. Como tales, contienen un deber ser ideal, todavía no contienen un deber ser relativo a las posibilidades fácticas y jurídicas». Sin embargo, 
AlEXY considera que «tiene todo el sentido referirse a los principios como mandatos de optimización», porque «[e]xiste una relación necesaria entre el deber ser ideal, es decir, el principio como tal, y el mandato de optimización, en cuanto regla. El deber ser ideal implica al mandato de optimización y viceversa. Son las dos caras de una misma moneda» (ibid., 110-112).

De acuerdo con lo anterior, los principios son ideales sujetos a una regla de optimización. ¿Cuál es esa regla? Esto es respondido por el siguiente postulado alexyano: «Entre la teoría de los principios y la máxima de la proporcionalidad existe una conexión [...que] no puede ser más estrecha: el carácter de principio implica la máxima de la proporcionalidad y esta implica aquella $($ sic). Que el carácter de principio implica la máxima de la proporcionalidad significa que la máxima de la proporcionalidad, con sus tres máximas parciales de la adecuación, necesidad (postulado del medio más benigno) y de la proporcionalidad en sentido estricto (el postulado de ponderación propiamente dicho) se infiere lógicamente del carácter de principio, es decir, es deducible de él». «De la máxima de proporcionalidad en sentido estricto», escribe ALEXY, «se sigue que los principios son mandatos de optimización con relación a las posibilidades jurídicas. En cambio, las máximas de la necesidad y de la adecuación se siguen del carácter de los principios como mandatos de optimización con relación a las posibilidades fácticas» (ibid., 111-113). Se colige, entonces, que la regla que manda optimizar la idealidad de los principios (y que se vincula conceptualmente con estos) es la máxima de la proporcionalidad, integrada por las aludidas tres máximas parciales.

Hoy por hoy, el uso del principio de proporcionalidad se ha extendido muy ampliamente como resultado de la propagación del Estado constitucional en la geografía del mundo y de la creación de tribunales supranacionales de derechos humanos, sin embargo, sus primeras formulaciones se remontan — cuando menos- al derecho público europeo del siglo XVIII (vid. BERNAL, 2007: 41 y ss.). Como nos recuerda BERNAL, la configuración del principio de proporcionalidad tendría su origen más claro en el contractualismo iusnaturalista de la época de la Ilustración, del que brotan dos presupuestos filosófico-políticos: «la idea de que la libertad se perpetúa en la sociedad civil como un bien inherente al individuo» y «la convicción de que la potestad estatal para intervenir en la libertad solo puede ejercerse en los casos necesarios y con la magnitud imprescindible para satisfacer las exigencias derivadas de los derechos de los demás y de los intereses esenciales de la comunidad». Así, en palabras de BERNAL, «[e]l disfrute de la libertad aparece entonces como la situación normal y la intervención estatal como una circunstancia excepcional, limitada en sus efectos a lo inexcusable», por lo que «[e]sta concepción del poder político fue el sustrato propicio para que se comenzara a exigir que las intervenciones estatales en la libertad individual fuesen proporcionadas» (ibid., 44-46). Por ello, la dogmática sobre el principio de proporcionalidad que se ha desarrollado en décadas recientes se enfoca principalmente en el uso de aquel principio por parte de los tribunales constitucionales cuando se trata de juzgar si una ley ha vulnerado derechos fundamentales. En ese contexto, como BERNAL escribe, «[e]1 principio de proporcionalidad cumple la función de estructurar el procedimiento interpretativo para la determinación del contenido de los derechos fundamentales que resulta vinculante para el Legislador y para la fundamentación de dicho contenido en las decisiones de control constitucional de las leyes» (ibid., 81). 
Si bien la teoría de los principios de ALEXY tiene como punto de partida el esquema dogmático de la máxima de proporcionalidad, cuyo contexto particular es el que se acaba de mencionar, la pretensión de la tesis de los principios como mandatos de optimización es la de proponer un esquema teórico general, inherente a la operatividad de los principios jurídicos como razones para la acción en cualquier contexto justificativo en que ellos intervengan. En esa medida, podemos afirmar que la máxima de la proporcionalidad es interpretada por ALEXY como una regla (o conjunto de reglas) configuradora(s) de un determinado esquema argumentativo complejo (o un conjunto de esquemas argumentativos simples, correspondientes a cada una de las máximas parciales), dentro del cual los principios actúan como razones para la acción ${ }^{1}$ dotadas de una peculiar operatividad justificativa. Es decir, la naturaleza de los principios estaría en que son susceptibles de un uso argumentativo típico: el que surge de la máxima de la proporcionalidad y que ALEXY elige llamar «optimización» (fáctica y jurídica).

\section{LA «OPTIMIZACIÓN» Y SU EQUIVOCIDAD}

No son claras las razones por las que AleXY eligió el término «optimización». Me atrevo a conjeturar que él buscó explicar la racionalidad de los principios jurídicos en el discurso jurídico-justificativo (y distinguirla de la de las reglas) estableciendo una analogía entre esta y la racionalidad económica basada en la eficiencia, es decir, en la óptima relación entre costos y beneficios. Con esta analogía, ALEXY posiblemente buscó mostrar el contraste entre las que para él —en palabras que usaría en un trabajo de 2003 - son las «dos dimensiones del razonamiento jurídico: una clasificatoria [classifying, conectada a la subsunción de reglas] y otra mensurativa [graduating, ligada a la ponderación de principios]» (ALEXY, 2003c: 448). El recurso a esa analogía pudo haberse inspirado en la constatación hecha por Bernard SCHLINK —en 1976- en el sentido de que la máxima de la necesidad representa una extrapolación al derecho del óptimo de Pareto (AleXY, 2002b: 164, nota 222; BeRNAL, 2007: 741) pues, como se verá más adelante, ALEXY traza explícitamente un nexo inevitable entre el óptimo de Pareto y la optimización fáctica de los principios. Debe constatarse, no obstante, que la anterior analogía fue mostrada de manera oscura en Teoría de los derechos fundamentales, lo que dejó a la «optimización» en la equivocidad, sin que las aclaraciones posteriores hayan logrado mitigarla; prueba de ello es que el término en cuestión ha sido entendido, al menos, en los tres sentidos que paso a caracterizar.

De acuerdo con una primera interpretación, la optimización de principios consistiría en la maximización de un estado de cosas. Con este sentido se correspondería la crítica de Atienza y Ruiz MANERo al concepto alexyano de principio. Aquellos autores, como se sabe, distinguen dos clases de principios: los «principios en sentido estricto» y las «directrices o normas programáticas». La diferencia estriba en que, si bien los dos tipos de norma presentan condiciones de aplicación abiertas (a diferencia de las reglas), los principios en sentido estricto tienen el consecuente cerrado, o sea,

1 Esto es así aunque AlEXY afirme que los principios son razones para la acción únicamente de manera indirecta, pues de modo directo serían, más bien, «razones para normas» (ALEXY, 2002b: 101). 
determinan la conducta prescrita, en tanto que las directrices configuran de manera abierta el modelo de conducta prescrito, dejándola indeterminada; por ello, los primeros son considerados normas de acción, mientras que las directrices, normas de fin; estas últimas ordenan o prohíben la consecución de objetivos determinados, de estados de cosas con ciertas característica (ATIENZA y RUIZ MANERO, 2004: 32 y ss.). «De ahí que», para los autores españoles, «a diferencia de lo que ocurre con los principios en sentido estricto, en la conducta gobernada por directrices no se trata de determinar la prevalencia de una u otra en relación con un determinado caso, sino de articular políticas capaces de lograr, en el mayor grado posible, la consecución de todos es[os] objetivos» (ibid., 33 y ss.) ${ }^{2}$. Pues bien, partiendo de la distinción expuesta, ATIENZA y RUIZ MANERO consideran que la caracterización de los principios como mandatos de optimización, según la cual los principios pueden ser cumplidos en diversos grados, es «distorsionadora» en relación con los principios en sentido estricto, porque un principio así «o se cumple o no se cumple», pero «perfectamente adecuada para dar cuenta de las directrices», donde «caben modalidades graduables de cumplimiento» (ibid., 31 y 33 y ss.). A esta primera interpretación corresponderían también las críticas que POSCHER ha dirigido a la teoría de AlEXY. En opinión de aquel, es equivocada la ya expuesta distinción alexyana entre, por un lado, los principios entendidos como mandatos a optimizar y, por otro, las reglas que operan como mandatos de optimización de los principios-objeto. Y lo sería porque «para reconstruir mandatos de optimización no se precisan mandatos que deban optimizarse, sino tan solo objetos que deban optimizarse. Los mandatos de optimización ordenan que se optimice algo, pero no ordenan que se optimicen mandatos» (POSCHER, 2011: 81) ${ }^{3}$.

Una segunda interpretación entendería que la optimización de los principios consiste en la fusión de estados de cosas a maximizar. En este sentido, PRIETO (en un trabajo publicado en 1992) parece asumir que, según ALEXY, los principios son «peculiares normas que pueden ser cumplidas en parte o hasta cierto punto», por lo que «al entrar en conflicto, admiten un cumplimiento simultáneo merced a la idea de mandato de optimización», pues «se considera posible conjugarlas en la mayor medida posible» (Prieto, 1992: 25 y 48) ${ }^{4}$. Una lectura similar parece tener SieCKMANn. Para él, la op-

2 Para ilustrar todo esto, los referidos dos autores, dan como ejemplo de principio en sentido estricto el expresado por el art. 14 de la Constitución española, que prohíbe la discriminación; y, de directriz, la expresada en el art. 51.1 de la misma Constitución, que ordena proteger mediante procedimientos eficaces la seguridad, la salud y los legítimos intereses económicos de los consumidores y usuarios (ATIENZA y RUIZ MANERO, 2006).

3 Poscher da un ejemplo: el art. 2, párr. 2, de la Ley Fundamental alemana «contendría el mandato de optimizar la integridad corporal y la salud, así como la vida. Pero la salud y la vida no son mandatos ni objetos normativos, sino objetos empíricos. [...] Los objetos de los mandatos de optimización adquieren fuerza normativa a través del mandato, incluido en los mandatos de optimización, de aumentar un objeto dentro de las posibilidades jurídicas y fácticas. [...] El sentido o dirección de la optimización no se encuentra tampoco predeterminado por el objeto del mandato, sino por el mandato de optimizar, el cual, por lo general, establece a través del contexto si el objeto, dentro de las posibilidades jurídicas y fácticas, debe ser maximizado o minimizado» (PosCHER, 2011: 81).

${ }_{4}^{4}$ Esto se apreciaría en un ejemplo que el mismo autor ofrece: «Imaginemos una cláusula testamentaria en la que un padre impone a su hijo como condición para acceder a los bienes (legítima y libre disposición) que no contraiga matrimonio con una mujer de religión judía». Para resolver el caso, en opinión de PRIETO, el juez podría renunciar sin más a la «idea de optimización», dando total preferencia a uno de los dos principios en juego: el de igualdad, que prohíbe la discriminación religiosa y racial, y el de autonomía de la voluntad, que protege las intenciones del testador. Sin embargo, en opinión del autor español, también sería posible que el juez (aplicando la idea de optimización) «intentase ponderar ambos principios, diciendo que el testamento es 
timización alexyana presupone que la satisfacción plena de tales principios no es posible, por lo que ellos «pueden ser realizados solo en una medida determinada, en cierto grado. El grado de cumplimiento corresponde — según SIECKMANN - a la relación entre el grado de cumplimiento alcanzado y la satisfacción plena». De este modo, las posibles soluciones a un caso «pueden contemplarse como combinaciones de los grados de satisfacción de los principios en colisión», frente ello, «puede preguntarse cuál combinación de los grados de satisfacción es óptima y por tanto est[á] definitivamente ordenada» (SIECKMANN, 2006: 163 y ss.).

Si bien se observa, esta segunda interpretación parece ser una consecuencia de la primera, pues el asumir la optimización como maximización de un estado de cosas implicaría el que debamos entender la optimización, también, como fusión de estados de cosas a maximizar, salvo que introduzcamos una - muy poco plausible — priorización lexicográfica entre los referidos estados de cosas. Dicha fusión consistiría en combinar, en un solo acto decisorio (simple o complejo), los distintos grados de realización de los estados de cosas perfilados por cada principio en juego ${ }^{5}$.

La tercera interpretación, en fin, es independiente de las dos anteriores y entiende la optimización jurídica de ALEXY como la maximización de la frecuencia de triunfos ponderativos de un determinado principio. PECZENIK parecería adscribir a esta comprensión cuando sostiene que, de acuerdo con la teoría de ALEXY, «[c] ada principio puede ser una razón prima facie para la acción. Pero pueden colisionar de manera tal que, por ejemplo, un incremento de respeto por la igualdad en el caso particular de que se trate pueda causar una disminución de la libertad y viceversa. Se necesita entonces un acto de sopesar y ponderar para escoger entre ellos en un caso individual». Por lo que «un principio alexyano nunca vence totalmente», pues «establece un ideal que puede ser llevado a efecto en una cierta clase de casos, más o menos extensa» (PECZENIK, 1992: 327-328; las cursivas son del texto). En la misma dirección se orientaría GARCíA FigUEROA en un libro publicado en 1998. Para él, «la aplicabilidad graduable propia de los principios puede predicarse de la intensidad de la aplicación (peso) o bien de la frecuencia de la aplicación (número de casos en que el principio se impone)». Lo explica de la siguiente manera: «La intensidad de un principio sería graduable en caso de aplicación parcial de su contenido. El principio se aplicaría más o menos intensamente. Por ejemplo, si en caso de que en un conflicto entre el principio de protección de la intimidad y el principio de libertad de información se

nulo por lo que se refiere a la legítima, pero válido en relación con los bienes de libre disposición» (PRIETO, 1992: 48 y ss.).

5 Esto tendría que ver con el tipo de «ponderación» que ATIENZA y RUIZ MANERO adscriben a las directrices, que consistiría «esencialmente en concretar un fin y/o sus formas de realización, teniendo en cuenta los límites marcados por otros fines y eligiendo medios que sean aptos para lograr el fin en cuestión, deteriorando lo menos posible el logro de otros fines también ordenados. [...] Es por ello», escriben los dos autores, «que la concreción de fines y de sus formas de realización que el cumplimiento de directrices entraña implica necesariamente, de un lado, una cierta priorización — aunque, desde luego, no lexicográfica一 de los diversos fines ordenados (a la que se podrían oponer otras priorizaciones igualmente admisibles) y, de otro, juicios, en la mayor parte de las ocasiones fuertemente controvertibles, sobre relaciones medio-fin» (ATIENZA y RUIZ MANERO, 2004: 37 y ss.). Este procedimiento racional contrastaría, en opinión de ATIENZA y RUIZ MANERO, con la clase de «ponderación» propia de los principios en sentido estricto, la que, más bien, consistiría «esencialmente en construir casos genéricos (conjuntos de propiedades) y determinar en cuáles de ellos prevalece un principio respecto de otro(s) principio(s) concurrente(s)» (ibid., 37), modalidad de ponderación que se correspondería, como luego se verá, con la «optimización jurídica» de ALEXY. 
alcanzara una solución intermedia consistente en satisfacer el derecho a la intimidad en 0,8 y el derecho a la información en 0,2» (GARCÍA FigUEROA, 1998: 190; la cursiva corresponde al texto). Mientras que «[1] a frecuencia de aplicación de los principios sería graduable en caso de su aplicación variable. El principio se aplicaría más o menos frecuentemente [..] Se produce, por usar la expresión de GUASTINI, una «jerarquía móvil»» (ibid., 191). Pues bien, sobre la base de la anterior distinción, GARCÍA FIGUEROA adscribe el concepto alexyano de principio a la optimización tomada como «frecuencia de aplicación» (ibid., 191 y ss.) y no a la entendida como «intensidad de la aplicación» (a la que, por cierto, parece corresponder el concepto de directriz). A partir de estos planteamientos, LOPERA distingue dos sentidos en los que, según ella, puede entenderse la idea de que los principios como mandatos de optimización «ordenan que algo se realice "en la mayor medida posible" ». «Como intensidad de la aplicación: predicable en el caso de aquellas normas que admiten un cumplimiento gradual o aproximativo (en una escala de 0 a 100 por 100), como sucede con la mayoría de los principios que prescriben la obtención de un estado de cosas (las directrices, en el lenguaje de ATIENZA y RUIZ MANERO)» Y «[c]omo frecuencia de la aplicación. Tal es el sentido que adquiere la exigencia de cumplimiento «en la mayor medida posible» respecto de aquellos principios cuyo objeto no admite una realización aproximativa («no matar», «no discriminar»), caso en el cual la exigencia que incorpora el mandato de optimización es una exigencia de cumplimiento «en el mayor número de ocasiones posibles». En este sentido», prosigue LOPERA, «cabe entender la graduabilidad incorporada a la noción de mandato de optimización en el caso de aquellos principios que prescriben la realización de acciones» (LOPERA, 2004: 231; las cursivas son del texto), es decir, los principios en sentido estricto de ATIEnZa y Ruiz MAnERo. Aunque LoPERA no es del todo clara en este punto, ella parece adjudicar al concepto alexyano de principio — como lo había hecho GARCía FIGUEROA — el sentido de optimización como «frecuencia de la aplicación».

La expuesta pluralidad de interpretaciones a que ha dado lugar la tesis de los mandatos de optimización es, como se dijo, una consecuencia de la equivocidad del término «optimización», lo que, teniendo en cuenta la centralidad que ALEXY concede a esta en su edificio teórico, viene a ser — digamos así- un aspecto subóptimo del mismo, debido a que la claridad conceptual de este se ve reducida dificultándose su crítica.

Esbozaré, enseguida, una reconstrucción del concepto alexyano de principio, teniendo como idea rectora la siguiente: la naturaleza de los principios viene dada por su conexión necesaria con ciertos modelos argumentativos típicos, los configurados por las reglas correspondientes a las tres máximas parciales de la proporcionalidad. Por ello, básicamente, me ocuparé de bocetar dichos modelos en torno a sus dos elementos (que para nuestros fines son) centrales: por un lado, los problemas argumentativos típicos que ellos presuponen, a los que debe responderse mediante la actividad argumentativa; y por otro, los roles operativos típicos atribuidos, en el marco de tal actividad, a los principios en cuanto razones para la acción ${ }^{6}$.

\footnotetext{
6 Sobre el concepto y las dimensiones de la argumentación jurídica, vid. ATIENZA, 2006 y 2013.
} 


\section{LA OPTIMIZACIÓN FÁCTICA O PARETIANA}

Como ya se dijo, ALEXY vincula conceptualmente la optimización fáctica con las máximas —o subprincipios- de la adecuación (o de la idoneidad) y de la necesidad. Para explicar la máxima de la adecuación, el autor alemán proporciona el siguiente ejemplo: «[s]upongamos que el legislador introduce la norma $N$ con la intención de mejorar la seguridad del Estado. $N$ infringe la libertad de expresión. La seguridad del Estado [.... la que identificaremos como principio P1]. La libertad de expresión [...a la que llamaremos principio P2]. Supongamos ahora que la norma $N$ no es adecuada para promover $P 1$, esto es, la seguridad del Estado, y sin embargo, infringe P2, o sea, la libertad de expresión. En este caso de inadecuación, existe la posibilidad fáctica de cumplir ambos principios conjuntamente en una mayor medida, declarando inválida $N$, que aceptando la validez de $N$. Aceptar la validez de $N$ no conlleva ninguna ganancia para P1 sino solo pérdidas para P2» (ALEXY, 1993: 32). La máxima de la necesidad, por su parte, puede explicarse retocando el ejemplo anterior: supongamos que se consideran las normas $N$ y $N$, ambas suficientemente adecuadas para promover la seguridad del Estado, P1; y que, de aquellas, $N>$ afecta menos la realización de la libertad de expresión, $P 2$, que $N$. En esta situación, $N$ no es necesaria para realizar $P 1$, porque $P 1$ puede ser cumplido con un costo menor.

ALEXY es enfático al sostener que si bien esas dos máximas «expresan la pretensión, contenida en el concepto de principio, de alcanzar la mayor realización posible de acuerdo con las posibilidades fácticas», esto «tiene muy poco que ver con un "punto máximo"» (AlEXY, 2002a: 27). Así, por lo que respecta a la máxima de la adecuación, el autor aclara que esta «no consiste en navegar hacia un punto máximo», sino que «tiene más bien el status de un criterio negativo [«establece un límite negativo» (ALEXY, 2003b: 62)], mediante el cual se puede detectar qué medios no son idóneos. Un criterio de esta naturaleza no lo fija todo, sino que únicamente excluye algo: los medios no idóneos [...], su función consiste en excluir lo no idóneo, sin que de este modo llegue a fijarlo todo» (ALEXY, 2002a: 28). En tal virtud, para nuestro autor, esa máxima «[n]o es otra cosa sino una expresión de la idea del óptimo de Pareto. Una posición puede ser mejorada sin perjudicar a otra» (ALEXY, 2011c: 13 y ss.). Y, en lo concerniente a la máxima de la necesidad, en palabras de ALEXY, esta «también es una expresión de la idea del óptimo de Pareto. A causa de la existencia de un medio más benigno e igualmente idóneo, puede mejorarse una posición sin que esto represente costes para la otra». Sin embargo, ha de tenerse en cuenta que, de manera similar a lo que ocurría con la máxima de la adecuación, según la máxima de la necesidad, «[a]l legislador no le está categóricamente prescrito adoptar el medio más benigno. Únicamente se afirma que si el legislador quiere perseguir su fin solo puede adoptar el medio más benigno, o un medio igualmente benigno o un medio un poco más benigno. No se trata de una optimización hacia ningún punto máximo, sino simplemente de la prohibición de sacrificios innecesarios» (ALEXY, 2002a: 29); esta máxima opera, entonces, «de manera negativa» (ALEXY, 2003b: 63 y ss.). Coherentemente con todo lo anterior, ALEXY sostiene: «Al haber delimitado el concepto de lo fáctico de esa manera, muchas cosas que en el lenguaje cotidiano designan lo fáctico - como por ejemplo lo económicamente deseable o ideas similares, o la socialización o la resocialización del hombre- entran, como fines, a la segunda categoría, la de las posibilidades jurídicas. Aquí, los aspectos 
culturales, morales y económicos, además de los legales, en todo caso juegan, a primera vista, un considerable papel» (ALEXY, 2003a: 92 y ss.).

La optimización fáctica, entonces, puede reconstruirse como un modelo argumentativo complejo, integrado por dos modelos simples con sendos problemas argumentativos. El primero es el esquema de la adecuación y su problema típico sería este: dada una medida, M1, adoptada con miras a satisfacer un principio que opera como fin, $\mathrm{P} 1^{\mathrm{f}}$, y bajo el presupuesto de que M1 socava el principio que frente al anterior opera como su restricción, $\mathrm{P} 2^{\mathrm{r}}$, ¿es M1 adecuado para la realización de P1 ${ }^{\text {f }}$ ? Y el segundo es el esquema de la necesidad, cuyo problema típico sería el siguiente: Dado un contexto en el que se consideran dos medidas, M1 y M2, ambas igualmente adecuadas para la realización de un principio que opera como $f i n, \mathrm{P} 1^{\mathrm{f}}$, y bajo el presupuesto de que $\mathrm{M} 1$ socava el principio que frente al anterior, opera como «su» restricción, $\mathrm{P} 2^{\mathrm{r}}$, ¿ंes M2 menos perjudicial que M1 (o nada perjudicial) para P2 $2^{\mathrm{r}}$ ? Los dos problemas señalados determinan que la argumentación desplegada en los modelos de la adecuación y de la necesidad tienen como propósito establecer si está justificado o no proscribir la adopción de la medida en cuestión por considerarse que es inadecuada y/o innecesaria para la consecución de un cierto principio que opera como $f n^{7}$, presuponiendo que tal medida es perjudicial para un principio que opera como restricción del primer principio.

Los dos principios en juego, como se observa, tienen operatividades asimétricas entre sí. El uno cumple un papel restrictivo, opera como razón para privar de justificación prima facie a ciertas acciones. El otro principio, en cambio, cumple el papel de fin, opera como razón para dotar de justificación prima facie a determinados cursos de acción. El primero de estos dos roles alude al comportamiento de los principios como límites negativos, conceptualmente próximos a las «restricciones laterales» (side constraints) de NOZICK (NOZICK, 1974: 28 y ss.), si bien ellas no tienen carácter prima facie sino definitivo. Esta faceta operacional de los principios es la que está en el foco de la optimización fáctica. Así, por lo que respecta al modelo de la adecuación, la focalidad operativa de $\mathrm{P} 2^{\mathrm{r}}$ está en que su socavamiento por parte de M1 es el presupuesto para que el problema de la adecuación tenga lugar: de no ser ese el caso, ni siquiera llegaría a plantearse la pregunta de si M1 es en alguna medida un medio adecuado para realizar $\mathrm{P}^{\mathrm{f}}$. Es decir, esta cuestión - la de la relación medio/fin - por sí sola es irrelevante para el modelo de la adecuación: argumentar sobre la inadecuación de M1 respecto de $\mathrm{P} 1^{\mathrm{f}}$ carece de pertinencia para ese modelo, a menos que previamente se haya argumentado que M1 socava P2r. Y, en lo concerniente al modelo de la necesidad, la focalidad operativa de $\mathrm{P} 2^{\mathrm{r}}$ reside, por un lado, en que aquí también se presupone que M1 socava $\mathrm{P}^{\mathrm{r}}$; y por otro, en que el problema de la necesidad requiere argumentar sobre la posibilidad o no de reducir el detrimento de $\mathrm{P} 2^{\mathrm{r}}$ mediante la elección, de entre los medios considerados en el contexto decisorio de referencia, de aquel que, siendo igualmente adecuado que $\mathrm{M} 1$ para realizar $\mathrm{P} 1^{\mathrm{f}}$, socave a $\mathrm{P} 2^{\mathrm{r}}$ en menor grado.

7 Nótese que prefiero distinguir entre principios que operan como fin y principios que operan como restricción, en lugar de emplear la distinción — que juzgo más o menos equivalente- de ATIENZA y RUIZ MANERO entre principios en sentido estricto y directrices. La razón principal de esto es que, a mi juicio, se da mejor cuenta de la construcción alexyana — lo que se verá al final de este trabajo, según aspiro— si, en lugar de distinguir entre dos tipos de principios, se distingue entre dos tipos de operatividad de los principios. 
Parece claro, entonces, que tanto en la adecuación como en la necesidad, la optimización consistiría en argumentar sobre la factibilidad o no de impedir o reducir el detrimento de los principios en cuanto razones restrictivas prima facie. Establecido así el sentido de la optimización fáctica, parecerían no tener cabida las interpretaciones que entienden la optimización alexyana como la maximización de estados de cosas, o como la fusión de estados de cosas a maximizar, lecturas originadas en la ya criticada equivocidad del término que nos ocupa. En efecto, el problema de la adecuación no implicaría en modo alguno la exigencia de diseñar y adoptar el medio que maximice la realización $\mathrm{P} 1^{\mathrm{f}}$; dicho problema ni siquiera implicaría que la realización del fin esté ordenada, pues bien podría estar prohibida o, incluso, permitida (vid. ALEXY, 2002a: 23 y ss). Y, por lo que respecta a la pregunta de la necesidad, esta no exigiría adoptar el medio más benigno de todos los fácticamente posibles, más allá del contexto decisorio de referencia. A este respecto, sin embargo, cabe hacer una observación: el modelo de la necesidad sería ajeno a toda maximización de estados de cosas debido a que introduce dos acotaciones. Por un lado, no se trataría de elegir el medio más benigno para $\mathrm{P} 2^{\mathrm{r}}$ independientemente del grado de realización que ese medio brinde a $\mathrm{P} 1^{\mathrm{f}}$, sino que debe serlo - al menos- en el mismo grado que M1. Y por otro, el universo de medios a considerar estaría limitado a los pertenecientes al contexto decisorio del que se trate. Por ejemplo, los que aparezcan en el proceso judicial de inconstitucionalidad de una determinada ley; de manera que el juez constitucional estaría vedado de considerar todos los medios que el legislador hipotéticamente hubiese podido prever. Ciertamente, si no existiera este freno, el modelo de la necesidad tomaría una deriva abiertamente maximizadora o, más exactamente, minimizadora de los detrimentos de $\mathrm{P}^{\mathrm{r}}$. Sin embargo, ALEXY no desarrolla los criterios para delimitar el conjunto de medidas aptas para ingresar en el escrutinio del decisor. Esta observación es importante porque solamente un desarrollo teórico en tal sentido disiparía del todo la idea de que la optimización fáctica tiene que ver con la maximización de estados de cosas.

En cualquier caso, como escribe ALEXY, la «optimización relativa a las posibilidades fácticas consiste en ahorrar costos que pueden ser evitados». Sin embargo, como él mismo agrega, «los costos devienen inevitables si los principios entran en conflicto. La ponderación entonces se hace necesaria» (ALEXY, 2011c: 15). Con lo cual pasamos al plano de la optimización jurídica.

\section{LA OPTIMIZACIÓN JURÍDICA O PONDERATIVA}

Ya se ha dicho que la optimización alexyana no se refiere únicamente a las posibilidades fácticas sino también a las posibilidades jurídicas. También hemos anticipado que estas últimas vienen dadas por los principios y reglas opuestas. Por tanto, la optimización jurídica de los principios (consistente — como ya se ha dicho—en la máxima de la proporcionalidad en sentido estricto) estaría referida a la solución de dos tipos de antinomias: principios-reglas y principios-principios. Dentro del primer tipo, al decir de AlEXY, cabe distinguir dos casos. En el primero, la regla en colisión es inderrotable, por lo que las posibilidades jurídicas del principio opuesto son nulas, vale decir, su optimización es inviable. AlEXY observa, no obstante, que en los ordenamientos jurídicos modernos, más bien, ocurre el segundo caso: las reglas suelen ser derrotables, 
por lo que el choque entre ellas y los principios se reconduciría a una colisión —puramente- de principios; entre, por un lado, el principio (o principios) opuestos a las reglas y, por otro, los principios formales y materiales que la respaldan (ALEXY, 2002b: 86, núm. 24). De ahí que — siempre según nuestro autor— «[1]a ponderación no [sea] más que la optimización relativa a principios contrapuestos» (ALEXY, 2005: 90) y, a la inversa, que «la optimización en relación con los principios que juegan en sentido contrario no consist[a] en nada diferente a la ponderación» (ALEXY, 2002a: 32). Consiguientemente, los conceptos de proporcionalidad en sentido estricto, optimización jurídica y ponderación mantienen entre sí equivalencia lógica (vid. ALEXY, 2011c: 19).

Arribamos, entonces, a una conclusión importante: en lo que atañe a las posibilidades jurídicas, el que los principios sean «mandatos a optimizar» no significa sino que ellos son mandatos a ponderar. Mucho de esto es captado por la —antes explorada - interpretación de la «optimización» de los principios como la maximización de la frecuencia de triunfos ponderativos, o sea, de su frecuencia de dominación en cuanto razones para actuar. Sin embargo, es preciso tener bien presente que la optimización de los principios no adscribe a estos una frecuencia de dominación estadística, sino, más bien, una frecuencia de dominación caso por caso $^{8}$ (lo que, por cierto, GARCÍA FigUEROA y LOPERA no perdían de vista). Esto significa que el número de ocasiones en que un cierto principio jurídico sea el dominante no depende de la elección de un agente obligado a maximizar esa frecuencia de dominación (como sucede, por ejemplo, con los deberes de beneficencia), sino que dicha frecuencia de dominación va a ser el resultado de ponderar correctamente un principio en cada caso que se presente. Aclarado esto, vemos que la noción de «frecuencia caso por caso» no es desatinada, pero no alcanza a explicar a fondo la optimización alexyana porque deja sin responder la pregunta de en qué sentido la ponderación de principios es una optimización que ocurre al interior de cada caso. Y, por ende, sin referencia al resultado agregativo (la frecuencia de dominación).

En adelante esbozaremos una reconstrucción de la optimización jurídica en cuanto modelo argumentativo complejo que, en palabras de ALEXY, «incluye[..] la ley de la colisión y la ley de la ponderación que ha encontrado su expresión en la fórmula del peso» (ALEXY, 2011b: 25).

\subsection{La ley de la colisión}

«La solución de la colisión [de principios] consiste», escribe ALEXY, «en que, teniendo en cuenta las circunstancias del caso, se establece una relación de precedencia condicionada. La determinación de la relación de precedencia condicionada consiste en que, tomando en cuenta el caso, se indican las condiciones bajo las cuales un principio precede al otro. Bajo otras condiciones, la cuestión de la precedencia puede ser solucionada inversamente» (ALEXY, 2002b, 92; las cursivas son del texto). De hecho, según dicho autor, la «muy difundida metáfora del peso» no significa sino que «[e]1 principio $P 1$ tiene, en un determinado caso concreto, un peso mayor que el principio

8 Sobre estas dos nociones de «frecuencia de dominación», predicables de las razones morales, vid. BAYON, 1991: 363 y ss. 
opuesto $P 2$ cuando existen razones suficientes para que $P 1$ preceda a $P 2$, bajo las condiciones $C$ dadas en el caso concreto» (ibid., 93).

ALEXY subraya, sin embargo, que «C es un presupuesto de un supuesto de hecho de una norma. [...] Así pues, de un enunciado de preferencia condicionada se sigue una regla que prescribe la consecuencia jurídica del principio que tiene preferencia cuando se dan las condiciones de preferencia» (ibid., 94). Todo esto es sintetizado por AlEXY en una regla a la que denomina ley de colisión, y que formula así: «Si el principio $P 1$, bajo las circunstancias $C$, precede al principio $P 2$ : (P1 P P2) $C$, y si de $P 1$ bajo las circunstancias $C$ resulta la consecuencia $R$, entonces vale la regla que contiene a $C$ como supuesto de hecho y a $R$ como consecuencia jurídica: $C \rightarrow R$ ». Y una «formulación algo menos técnica», según nuestro autor, rezaría: «las condiciones bajo las cuales un principio precede a otro constituyen el supuesto de hecho de una regla que expresa la consecuencia jurídica del principio precedente» (ibid.). La ley de colisión, por consiguiente, bien podríamos formalizarla así:

(1) $($ P1 P P2) $C$

(2) $(P 1 \rightarrow R) C$

(3) $C \rightarrow R$ (vid. ibid., 158)

Podríamos saturar este esquema inferencial explicitando su punto de partida: la colisión misma. De manera que, retocando la ley de colisión de ALEXY, llegaríamos a esta reformulación: $O$ bien, bajo las circunstancias $C$, el principio $P 1$ precede a P2, y entonces vale la regla $C \rightarrow R$, o bien, bajo las circunstancias $C$, el principio $P 2$ precede a $P 1$, y entonces vale la regla $C \rightarrow \neg R$; bajo las circunstancias $C$, el principio $P 1$ precede a $P 2$; por tanto, vale la regla $C \rightarrow R$. Esto nos conduciría a una formalización completa de la ley de colisión, así:

(1') $\quad((P 1 \mathrm{P} P 2) C \rightarrow(C \rightarrow R)) \underline{\vee}((P 2 \mathrm{P} P 1) C \rightarrow(C \rightarrow \neg R))$

(2') $\quad(P 1$ P P2) $C$

(3') $\quad C \rightarrow R^{9}$

9 Un esbozo preliminar de este esquema formal elaboré en LOZADA, 2012, y es semejante al esquema formal que ATIENZA, diseña para dar cuenta de la ponderación, a saber (ATIENZA, 2006: 171):

- En la situación concreta $S$, el principio $P 1$ y el principio $P 2$ - que tienen condiciones de aplicación abiertas - establecen exigencias normativas contrapuestas (por ejemplo, permitido $q$ y prohibido $q$ ).

- En la situación concreta S, dadas las circunstancias C, un principio prevalece sobre el otro (por ejemplo, P1 sobre $P 2)$.

- Por tanto, en esa situación y dadas esas circunstancias, está justificado dictar una norma que establece que si p (un conjunto de propiedades que incluye las derivadas de las circunstancias C), entonces está prohibido $q)($ ). siguiente:

Esquema que, al decir de su autor, sería susceptible de ser expresado mediante la formalización lógica

$($ ) $\rightarrow P q[P 1]$

$($ ) $\rightarrow P b q[P 2]$

$S / P q \wedge P h q$

$S, C / P 2>P 1$

$S, C / J(p \rightarrow P h q)$

[...] La situación S se refiere a una situación concreta definida por todas las circunstancias del caso de que se trate; $\mathrm{p}$ representa, abreviadamente, un conjunto de propiedades seleccionadas por el juez para confeccionar el caso abstracto (o supuesto de hecho) de la norma que se pretende crear; y $\mathrm{C}$ son las circunstancias que sirven de justificación para configurar una tal norma. 
Como puede apreciarse, la conclusión (3) sería idéntica a la (3'), la premisa (1) lo sería a la (2'); y la premisa (2) devendría un fragmento de la (2'). Esta formalización completa de la ley de colisión ofrece la ventaja de clarificar el modelo complejo al que ella da lugar, el que llamaremos modelo de la optimización jurídica o de la ponderación en sentido amplio. Dentro de este, las dos premisas de la ley de la colisión y su correspondiente conclusión conforman un razonamiento deductivo dirigido a responder al que podría denominarse problema de la ponderación en sentido amplio, mediante la afirmación de la validez o la invalidez de una regla, C $\rightarrow$ R1. No obstante, más allá de ese razonamiento deductivo (que vendría a ser la justificación interna de la ponderación en sentido amplio), salta a la vista que las premisas (1') y (2') requieren sendas fundamentaciones (constitutivas de la justificación externa de las premisas que componen la ponderación en sentido amplio) que van a dar lugar, respectivamente, a los siguientes modelos argumentativos simples: el modelo de la colisión y el modelo de la ponderación en sentido estricto o del balanceo (a este último me referiré en adelante simplemente como «modelo de la ponderación»)

La premisa (1') expresa con mayor precisión lo que es una colisión de principios: esta se da cuando, por un lado, existen dos principios - o conjuntos de principiostales que, en un determinado caso, cada uno proyecta sendas plasmaciones normativas con forma de reglas; y por otro lado, cuando estas son lógicamente incompatibles entre sí. Esta premisa, por consiguiente, explicita — como ya se dijo— la colisión de principios en cuanto presupuesto de la ponderación, pero además aclara y precisa aquello en lo que esto último consiste: en un impasse deliberativo de estructura binaria. El que se caracteriza por la concurrencia de dos elementos, a saber: i) la inminencia de tomar una decisión normativa binaria entre reglas mutuamente excluyentes: $(C \rightarrow R)$ $\underline{\vee}(C \rightarrow \neg R)$. Y ii) la necesidad de resolver, previamente, una tensión valorativa binaria entre principios que respaldan tales reglas, tensión que subyace a, y es el origen de, la decisión normativa binaria: (P1 P P2) (P2 P P1). Nótese que esta estructura binaria parece descartar la plausibilidad de interpretar la optimización jurídica de los principios como la fusión de estados de cosas a maximizar (y, por cierto, como maximización de estados de cosas): si P1 y P2 colisionan, o prevalece el uno o prevalece el otro, no tendría sentido tratar de combinarlos. Pero la estructura binaria del impasse deliberativo que venimos analizando muestra algo más. La conexión entre la tensión normativa binaria y la tensión valorativa binaria viene dada por la relación cruzada entre cada una de las reglas mutuamente incompatibles y el principio opuesto: $(C \rightarrow R)$ y $P 2$, por

\footnotetext{
Se puede apreciar que esta esquematización formal es básicamente equivalente a la de ALEXY, salvo en dos aspectos que pueden ser relevantes en ciertos contextos de análisis: En primer lugar, en que la primera premisa del esquema alexyano (antes de la reformulación que proponemos) se correspondería con tres de las premisas que componen la propuesta de ATIENZA. Las dos primeras premisas expresan respectivamente la presencia de los principios $P 1$ y $P 2$ y el carácter abierto de sus condiciones de aplicación, representadas con «( )»; y la tercera premisa explicita la colisión de los principios, es decir, justamente lo que echamos en falta en la construcción de ALEXY: $S / P q \wedge P h q$. Y, en segundo lugar, en que el esquema formal de ATIENZA es más fino a la hora de reconstruir el proceso de configuración del supuesto de hecho de la regla resultante de la ponderación, pues distingue y articula la situación concreta del caso, $-\mathrm{S}-$, las condiciones normativamente relevantes para la configuración de la norma, -C-, y el supuesto de hecho de esta -p-, mientras que en ALEXY esos tres términos están englobados, indiferenciadamente, en C; lo que hace que aquella formalización sea de mayor utilidad que esta cuando se trate de problematizar el tramo argumentativo que va desde la totalidad de circunstancias que el caso concreto presenta hasta la configuración del supuesto de hecho de la norma.
} 
un lado, y $(C \rightarrow \neg R)$ y $P 1$, por otro. Esta es una relación de detrimento, ya que cada una de las reglas socava el principio opuesto. Esta relación cruzada, que será aclarada enseguida, constituye el nervio mismo de la colisión de principios.

De lo anterior se sigue que, el modelo de la colisión, atinente a la premisa (1') de la ponderación en sentido amplio, presupone el que podemos denominar problema de los detrimentos alternativos, que puede formularse así: ¿por qué la afirmación de la validez de $C \rightarrow R$ implica el menoscabo de $P 2$, y la de $C \rightarrow \neg R$, el de $P 1$ ? Si retomamos en este punto la nomenclatura usada en el examen de la optimización fáctica, y reparamos en que la regla $C \rightarrow R$ equivale a la medida M1 y que la regla $C \rightarrow \neg R$ equivale a la medida $\neg \mathrm{M} 1$, podemos analizar este problema dividiéndolo en dos. Por un lado, el subproblema del detrimento de la restricción: ¿por qué M1 socavaP2 ${ }^{\mathrm{r}}$ ?; y, por otro, el subproblema del detrimento del fin: ¿por qué $\neg \mathrm{M} 1$ socava P1? Recordando lo expuesto hace varios párrafos, podemos constatar que la respuesta afirmativa al subproblema del detrimento de la restricción era el presupuesto tanto del problema de la adecuación como del problema de la necesidad; esto revela que este subproblema tiene prioridad lógica respecto del resto de problemas argumentativos, es el eje que vertebra la optimización fáctica con la jurídica.

Esto permite precisar que, en el modelo de la colisión, los principios operan como razones para juicios sobre detrimentos valorativos (es el caso tanto de $\mathrm{P} 1^{\mathrm{f}}$ como de $\mathrm{P} 2^{\mathrm{r}}$ respecto de $\neg \mathrm{M}$ y $\mathrm{M}$, respectivamente). Sin embargo, es obvio que tales juicios están vinculados a juicios cuyas razones son principios que operan como fines (es el caso de $\mathrm{P} 1^{\mathrm{f}}$ respecto de $\mathrm{M}$ ) o que operan como restricciones (es el caso de $\mathrm{P} 2^{\mathrm{r}}$ respecto de $\neg \mathrm{M}$ ). En torno a estos dos tipos de rol operativo de los principios como fines y como restricciones, cabe hacer dos observaciones. Por un lado (el de P2 ${ }^{\text {r }}$, ALEXY no clarifica cuál es la índole de la relación que hay entre $\neg \mathrm{M} 1$ y P2 ${ }^{\mathrm{r}}$, o sea, entre la no adopción del medio adecuado (o bien, de la regla equivalente) para la realización del fin y la restricción de este. ¿Se trata de una relación conceptual?, ¿causal?, ¿teleológica? Por otro lado (el de $\mathrm{P} 1^{\mathrm{f}}$, si bien nos queda claro que la relación entre M1 y P1 es de carácter teleológico, surge aquí un interrogante: ¿el grado de realización de P1 $1^{\mathrm{f}}$ producido por M1 (es decir, por $C \rightarrow R$ ) es idéntico al grado de detrimento de $\mathrm{P} 1^{\mathrm{f}}$ originado por $\neg \mathrm{M} 1$ ? ALEXY parece dar por hecho que no lo son: en la Teoría de los derechos fundamentales, al tratar sobre los derechos de protección advirtió que la peculiaridad de estos «resulta de dos cosas. Por una parte, los medios de protección no pueden ser clasificados sin más en dos clases, eficaces y no eficaces. Existen medios de protección más o menos eficaces. Por otra, los deberes de protección tienen el carácter de principios, es decir, exigen una protección lo más amplia posible con relación a las posibilidades fácticas y jurídicas, lo que significa que pueden entrar en colisión con otros principios» (ALEXY, 2002b: 448). Es decir, ALEXY parece distinguir en ciertos principios (como los relativos a derechos de protección) la dimensión de la eficacia y la dimensión de su optimización. Esta distinción parece reflejarse en Sobre la estructura de los derechos fundamentales de protección (Zur Struktur der Grundrechte auf Schutz), un trabajo de 2007, donde ALEXY diferencia entre dos variables, el «grado de protección» proveniente de las medidas realizadoras de un derecho de protección, y la «intensidad de la afectación» provocada a un derecho de protección por un principio opuesto (ALEXY, 2011c: 128 y ss.). Nuestro autor no muestra de manera cabal el porqué de esa distinción ni, en todo caso, cuáles serían las relaciones justificativas entre ambas variables. Este par de obser- 
vaciones muestran que el modelo de la colisión no ha recibido por parte de ALEXY un desarrollo completo.

\subsection{La ley de la ponderación}

Ahora bien, por lo que respecta al modelo de la ponderación, concerniente a la premisa (2') de la ponderación en sentido amplio, este plantea el problema de la precedencia condicionada, que puede formularse así, ¿cuál de los principios en colisión es el dominante en el caso concreto de que se trata? La argumentación llamada a responder esta pregunta, según ALEXY, debe seguir una regla a la que él denomina «ley de la ponderación», y que reza: «[c]uanto mayor es el grado de la no satisfacción o afectación de un principio, tanto mayor tiene que ser la importancia de la satisfacción del otro» (AlEXY, 2002b: 161). Si, como antes se dijo, la ponderación consiste en la optimización de los principios en relación con las posibilidades jurídicas, «[1]a ley de la ponderación dice en qué consiste esta relación»: «La medida permitida de no satisfacción o de afectación de uno de los principios depende del grado de importancia de la satisfacción del otro» (ibid.); lo que hace — siempre según ALEXY — que «la ponderación no [sea] un procedimiento en el cual un bien es obtenido con "excesivo apresuramiento" a costa de otro» y que «respond[a] al llamado principio de la concordancia práctica» (ALEXY, 2002b: 166 y ss.). En suma, y como se anticipó, «la optimización en relación con los principios que juegan en sentido contrario no consiste en nada diferente a la ponderación» (ALEXY, 2002a: 32).

Pues bien, «saber qué es la ponderación presupone», escribe ALEXY, «conocer su estructura. La ley de la ponderación indica que la ponderación puede ser fragmentada en tres etapas. La primera etapa establece los grados de insatisfacción o detrimento de un primer principio. Esta fase viene seguida por una segunda etapa, en la que se establece la importancia de satisfacer el principio opuesto. Finalmente, en la tercera etapa se establece si la importancia de satisfacer el segundo principio justifica el detrimento o la insatisfacción del primer principio» (ALEXY, 2005: 92 y ss.). ALEXY ha tratado de ilustrar esta «estructura de la ponderación» ${ }^{10}$ de dos maneras: la primera, la menos famosa, llevada a cabo en la Teoría de los derechos fundamentales, empleaba la metáfora microeconómica de la curva de indiferencia ${ }^{11}$, aunque el autor se encargó de advertir que si bien «[1] as curvas de indiferencia aclaran las ideas que se encuentran detrás de la

10 Como se sabe, en la literatura sobre la materia, se ha difundido la también denominada «estructura de la ponderación» integrada por la ley de la ponderación, la fórmula del peso y las reglas sobre la carga de la argumentación. Conviene recordar, sin embargo, que esta presentación no fue diseñada, tal cual, por ALEXY, sino por C. BERNAL en su conocido artículo Estructura y límites de la ponderación, de 2003. En mi opinión, esa reconstrucción de BERNAL, por un lado, completa el esquema alexyano, por cuanto explicita la presencia necesaria de cargas argumentativas en la ponderación; sin embargo, por otro lado, torna redundante el esquema alexyano al introducir en él la representación metafórica de la fórmula del peso, conjuntando en la estructura de la ponderación lo que esta es y la ilustración de lo que ella es.

11 ALEXY empieza por recordar que «[u]na curva de indiferencia es un medio para la representación de las relaciones de sustitución de bienes. Supongamos que a está tanto a favor de la libertad de prensa como de la seguridad externa y que está dispuesto a aceptar, en aras de un cierto aumento de la libertad de prensa, una cierta reducción de la seguridad externa y, por un cierto aumento de la seguridad externa, una cierta reducción de la libertad de prensa. Las situaciones que $a$ juzga como igualmente buenas o indiferentes pueden ser anotadas como puntos en una curva: 
ponderación [..., estas] no ofrecen un procedimiento definitivo de decisión» (ALEXY, 2002b: 163 y ss.). AlEXY pensaba que esta forma «indiferencialista» de representar la ponderación también es útil para representar la estructura del examen de necesidad con ayuda del criterio del óptimo de Pareto, por lo que la imagen de las curvas de indiferencia parecen haber estado en el origen de la tesis de los principios como mandatos de optimización (ALEXY, 2002b: 164, nota 222). Sin embargo, la segunda forma de ilustrar la ponderación, la más famosa hoy en día, es la metáfora matemática de la fórmula del peso, expuesta en el Epílogo. No entraré, aquí, en la exposición de esta bien conocida fórmula. Basta, para nuestros fines, con anotar que con ella ALEXY busca mostrar que así como «la subsunción es una regla que puede expresarse mediante un esquema deductivo llamado «justificación interna»; el cual se construye con la ayuda de las lógicas proposicional, de predicados y deóntica», la fórmula del peso viene a ser la «contraparte de este sistema deductivo» (ALEXY, 2005: 94): «Un esquema que funciona de acuerdo con las reglas de la aritmética» (ALEXY, 2003c: 448). Si bien AlEXY advierte que «[1] as premisas reales de la fórmula del peso no son números sino juicios acerca de los grados de interferencia, la importancia de los pesos abstractos y los grados de seguridad [relativos a los principios en juego]» (ibid., 448), no deja de tener razón ATIENZA cuando considera que el excesivo refinamiento de la fórmula constituye un error retórico, pues ha llevado a pensar a muchos que la clave de la argumentación está en la fórmula en sí, incurriendo así en lo que VAZ FERREIRA identificaba como falacia de falsa imprecisión (vid. ATIENZA, 2010: 50; ibid., 2014b: $\$ 2$ ). No obstante, puesto que toda disciplina matemática es una teoría deductiva (TARSKI, 1977: 152), el uso metafórico de la fórmula del peso resalta que la ponderación, al igual que la sub-

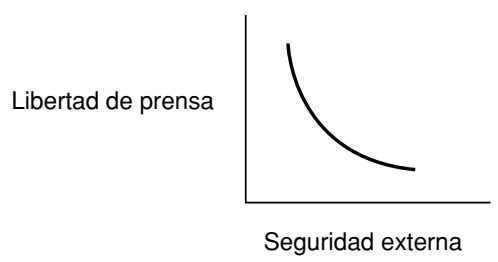

Este tipo de curvas de indiferencia responden a la ley de la decreciente tasa marginal de la sustitución. Si disminuye la libertad de prensa, se requieren aumentos cada vez mayores de la seguridad externa, a fin de compensar una disminución aún mayor de la libertad de prensa, y viceversa» (ALEXY, 2002b: 161 y ss.).

ALEXY aclara, sin embargo, que la curva describe el comportamiento de preferencias subjetivas de un cierto agente, mientras que para representar cómo opera la ley de la ponderación es preciso asumir a esta como «una regla que prescribe el establecimiento de curvas de indiferencias correctas (importancias relativas correctas). [...Así]:

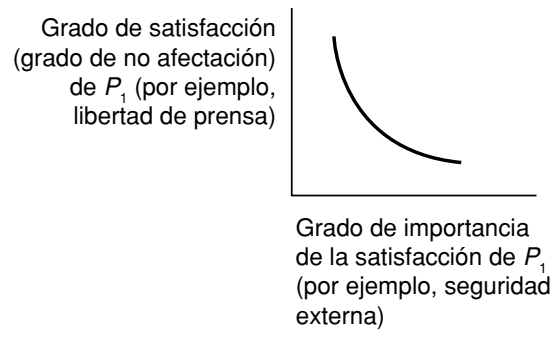

» (ibid., 163). 
sunción, tiene un andamiaje deductivo que deviene la justificación interna de la que antes hemos identificado como premisa (2'), es decir, del modelo de la ponderación ${ }^{12}$.

La presentación de la fórmula del peso, adicionalmente, ha sido la ocasión para que AlEXY dejara en claro que si bien la ley de la colisión conduce a hablar, por un lado, de la intensidad de la «intervención» en un primer principio $(P 1)$, y por otro, del grado de «importancia» de la satisfacción de un segundo principio $(P 2)$, este grado de «importancia» equivale conceptualmente a la «intensidad de la intervención que origina la no intervención» en el primer principio (ALEXY, 2002a: 39) ${ }^{13}$. Esto quiere decir que, en el marco del modelo de la ponderación, la operatividad de los principios no dice relación alguna con si ellos cumplen el papel de fines o de restricciones; de hecho, cuando ellos son balanceados entre sí, no operan ni como lo uno ni como lo otro. ¿En qué radica, entonces, la operatividad de los principios en el contexto de la ponderación?

Como se anticipó en supra $\mathbb{1}$, la idea de mandatos de optimización consiste, según AleXY, en que estos «pueden ser denominados como un "deber ser ideal" o simplemente pueden entenderse como "ideales"», es decir, «todavía no contienen un deber ser relativo a las posibilidades fácticas y jurídicas» (ALEXY, 2003d: 108 y ss.). En un trabajo de 2009, Deber ideal (Ideales Sollen), ALEXY introdujo el «operador de mandato indexado» «Oi» para expresar que la «vinculación necesaria entre el deber ideal y la optimización [...] se pone de manifiesto a través de la equivalencia [...]

${ }^{12}$ La teoría de la argumentación jurídica de ATIENZA — uno de cuyos antecedentes fuera Las piezas del derecho — escrita conjuntamente por ATIENZA y RUIZ MANERO en 1991- ha contribuido en mucho a mejorar el entendimiento de la ponderación, la propia del modelo alexyano y en general. Sobre tales contribuciones, véanse ATIENZA, 2006; 2010; 2013 y 2014b. En varios aspectos (que serían muy difíciles de inventariar) las ideas que presento aquí son tributarias de las de ATIENZA; particularmente, las de un trabajo suyo de hace un par de años, Ponderación y sentido común jurídico, donde sintéticamente reconstruye la ponderación en estos términos: «La ponderación es un procedimiento argumentativo en el que pueden distinguirse dos pasos. En el primero se pasa del nivel de los principios al de las reglas: se crea, por tanto, una nueva regla no existente anteriormente en el sistema de que se trate. Luego, en un segundo paso, se parte de la regla creada y se subsume en ella el caso a resolver. Lo que podría llamarse la "justificación interna" de ese primer paso es un razonamiento con dos premisas. En la primera se constata simplemente que, en relación con un determinado caso, existen dos principios (o conjuntos de principios) aplicables, cada uno de los cuales llevaría a resolver el caso en sentidos entre sí incompatibles: por ejemplo, el principio de libertad de expresión, a considerar permitida tal tipo de conducta; y el principio de respeto a la intimidad, a considerarla prohibida. En la segunda premisa se establece que, dadas tales y cuales circunstancias que concurren en el caso, uno de los dos principios (por ejemplo, el de libertad de expresión) derrota al otro, tiene un mayor peso. Y la conclusión vendría a ser una regla general que enlaza las anteriores circunstancias con la consecuencia jurídica del principio prevaleciente: si se dan las circunstancias X, Y y Z, entonces la conducta C está permitida. Naturalmente, la dificultad de ese razonamiento radica en la segunda premisa, y aquí es precisamente donde se sitúa la famosa «fórmula del peso» ideada por R. ALEXY que vendría a ser, por tanto, la "justificación externa" de esa segunda premisa. Como todo el mundo sabe a estas alturas, lo que viene a decir ALEXY es que, cuando se producen choques entre principios (lo que para él equivale a decir, entre derechos) esos conflictos deben resolverse aplicando el principio de proporcionalidad que, a su vez, consta de tres subprincipios: el de idoneidad, el de necesidad y el de proporcionalidad en sentido estricto; este último, a su vez, consta de tres elementos: la ley de la ponderación, la formula del peso y las cargas de la argumentación. La ley de la ponderación se concreta, a través de tres variables, en la fórmula del peso, siendo esas variables el grado de afectación de los principios en el caso concreto, el peso abstracto de los principios relevantes y la seguridad de las apreciaciones empíricas. Si existe un empate (el peso de los principios es idéntico), entonces es cuando entran en juego las reglas sobre la carga de la argumentación: por ejemplo, la que establece una prioridad a favor de la libertad, o a favor del criterio del legislador (la deferencia al legislador)» (ATIENZA, 2014b: $\$ 2$ ).

13 Sobre cómo esto ha repercutido en la notación usada en la fórmula del peso, vid. ALEXY, 2007. 
OOptOp $p \leftrightarrow$ Oip» (AleXY, 2011b: 25). Esto quiere decir que la proposición «es obligatorio optimizar que es obligatorio que p» (principio como mandato a optimizar) equivale a la proposición «es obligatorio idealmente que $p$ » (principio como mandato ideal). De aquí se sigue, en palabras de ALEXY, que «[e]l deber ideal, expresado a través del operador de mandato indexado, es un deber genuino que en caso de conflicto implica un deber concreto, el cual se debe medir todavía de acuerdo con las posibilidades de este mundo, tal como este es. El deber ideal abstracto es una razón para este deber concreto» (ibid., 22). Es por ello que si, como resultado del balanceo, el deber ideal no puede tornarse concreto, eso supone un detrimento del ideal en cuestión. En tal virtud, como lo indica la ley de ponderación (e ilustra la fórmula del peso), los principios operan como razones para establecer juicios comparativos sobre la intensidad de los detrimentos. Operan, entonces, como bienes o valores morales, o sea, como criterios para la formulación de los juicios evaluativos suficientes y necesarios para responder correctamente al problema de la precedencia condicionada. Así es posible comprender de mejor manera por qué el concepto de principio de ALEXY es, en realidad, un concepto de principio válido (ALEXY, 2002c: 105): la noción de principio se identifica con la de valor ${ }^{14}$, de manera que así como no cabe hablar de razones operativas para la acción consistentes en valores inválidos, tampoco cabría hablar de razones operativas para la acción que consistan en principios inválidos.

Esta comprensión alexyana de la ponderación, como se sabe, ha sido criticada por HABERMAS porque - en opinión de este- «los derechos fundamentales cobran un sentido distinto según se los entienda en el sentido de DwORKIN como principios jurídicos deontológicos, o en el sentido de AlEXY como bienes jurídicos optimizables. En tanto que normas regulan una materia en interés de todos por igual; en tanto que valores constituyen, en la configuración que forman con otros valores, un orden simbólico en el que se expresan la identidad y forma de vida de una comunidad jurídica particular [...]. La transformación conceptual de los derechos fundamentales en valores fundamentales», prosigue HABERMAS, «significa un enmascaramiento teleológico de los derechos, que no contribuye por cierto a percatarse de la circunstancia de que, en los contextos de fundamentación, las normas y los valores desempeñan papeles distintos en lo tocante a la lógica de la argumentación»; con lo cual «desaparecen esos cortafuegos que con la comprensión deontológica de las normas y principios jurídicos quedan introducidos en el discurso jurídico» (HABERMAS, 2005: 329 y ss., y 332). No voy a entrar aquí, desde luego, en el examen de esta polémica entre HABERMAS y ALEXY. Únicamente me interesa sostener que este último tiene razón cuando, en contestación a la reseñada crítica, sostiene que esta presupone un «concepto fuerte [del carácter deontológico de los derechos] según el cual lo debido es absoluto y universal de tal modo que pretende ser bueno para todos de igual medida [...; concepto que] presenta problemas cuando se trata de normas jurídicas» (ALEXY, 2003d: 125), pues algunas de ellas — como se sabe- son apenas prima facie. Y también tiene razón cuando agrega que «la ponderación y la optimización ligada a ella no son incompatibles con la idea de corrección, sino que incluso esta idea las exige» (ibid., 127): en la ponderación

14 Este es el sustrato de algo que ALEXY ha repetido con insistencia, y ha bautizado como «integridad del principio en retroceso»: «Si el principio es derrotado en la ponderación, no puede justificar un deber definitivo. Sin embargo, el principio continúa implicando el deber ideal a pesar de la derrota» (ALEXY, 2011b: 27). 
se trata de fundamentar, escribe ALEXY, «juicios de proporcionalidad», los que «como todos, enarbolan una pretensión de corrección y esta tiene como razones los juicios de grados de intensidad sobre los que se apoya», esto «se corrobora si no solo se mira la relación entre los juicios sobre grados de intensidad y el juicio de proporcionalidad, sino por otra parte también la que hay entre cada uno de los primeros y las razones que se enuncian -igualmente en un próximo nivel— para justificarlos» (ALEXY, 2009: 12). De manera que el modelo de la ponderación tiene a los principios operando como bienes o valores, o sea, como razones para evaluar los grados de intensidad de los detrimentos; pero estos constituyen, a su vez, razones para la corrección, es decir, para la no desproporción del juicio de precedencia condicionado y, en últimas, de la regla que culmina el procedimiento de ponderación en sentido amplio (y que es la razón operativa inmediata para la decisión del caso).

Si dejáramos de lado la terminología de AlEXY (innecesariamente alambicada, pero en la que hemos debido entrar para satisfacer nuestros fines reconstructivos), veríamos que la noción de ponderación simplemente representa el nivel más básico de la racionalidad práctica; pues se corresponde con el contenido del principio práctico P1 formulado por RAZ: «Es siempre el caso que se debe, todas las cosas consideradas, hacer lo que se debe hacer sobre la base del balance de razones» (RAZ, 1991: 41). De ahí que, según ha mostrado ATIENZA, los distintos elementos del modelo de la ponderación no consistirían en otra cosa que en «tópicos, puntos de partida, comúnmente aceptados» pertenecientes a un «sentido común jurídico» (ATIENZA, 2014: $\mathbb{\$} 3$ ). Si la abordamos de manera gruesa, la ponderación parece tener, inclusive, la fisonomía simple de la máxima del mal menor ${ }^{15}$, cuya estela histórica, como se sabe, es muy extensa: Platón, en el Protágoras, la exponía así: «cuando uno se vea obligado a escoger entre dos males, nadie elegirá el mayor, si le es posible elegir el menor» (PLATÓN, 1981, \$358d). ARISTÓTELES afirmaba que «el mal menor es también, en cierto modo, un bien» (ARISTÓTELES, 1993: $\$ 1129 \mathrm{~b}, 5$ ), y que «el mal menor, comparado con el mayor, se considera un bien, ya que el mal menor se prefiere al mayor, y lo preferible es un bien, y cuanto más preferible, mayor» (ibid., 1131b, 30). CICERÓN escribía que «de los males el menor debe ser» (CICERÓN, 1877, III: 1.9 y 28.6). Y TOMÁs DE AQUINO ofrecía esta formulación general: «Cuando es forzoso escoger entre dos cosas, en cada una de las cuales hay peligro, debe elegirse aquella de la que menos mal se sigue» (AQUiNO, 1998: 1. 1, c. 6); y en línea con los escritos aristotélicos, señalaba: «el mal menor constituye un bien en comparación con el mal mayor porque el mal menor es más elegible que el mal mayor. Cada uno elige basado en el bien. Y por eso lo más elegible constituye un bien mayor» [AQUinO, 1997 (5), 1. 5, n. 9]. Finalmente, MAQUIAVELO aconsejaba: «Nunca crea un Estado tomar decisiones con total seguridad; piense más bien que siempre se hallará en terrenos movedizos, pues forma parte del orden de las cosas que siempre que se busque evitar un inconveniente acabe cayéndose en otro; mas la prudencia consiste en saber reconocer la índole de los inconvenientes, y adoptar el menos malo como bueno» (MAQUIAVELO, 2011: 75).

Ahora bien, sobre la relación entre principios y valores, puesta sobre la mesa por todo lo que se ha expuesto, ALEXY manifiesta que la diferencia entre ambos conceptos

\footnotetext{
15 Un apunte en este sentido, vid. en AguiLÓ, 2015: 125.
} 
«se reduce $[. .$.$] a un punto. Lo que en el modelo de los valores es prima facie lo mejor$ es, en el modelo de los principios, prima facie debido; y lo que en el modelo de los valores es definitivamente lo mejor es, en el modelo de los principios, definitivamente debido». Así pues, concluye, «los principios y los valores se diferencian solo en virtud de su carácter deontológico y axiológico respectivamente» (ALEXY, 2002b: 147). «Esto muestra con claridad», en opinión de nuestro autor, «que el problema de las relaciones de prioridad entre principios se corresponde con el problema de una jerarquía de valores» (AlEXY, 1988: 145). AleXY elige usar el modelo de los principios dando, para ello, tres razones. En primer lugar, porque «[e]n el derecho, de lo que se trata es de qué es lo debido» y en el modelo de los principios «se expresa claramente el carácter de deber ser» (ALEXY, 2002b: 147). En segundo lugar, porque «no existe dificultad alguna en pasar de la constatación de que una determinada solución es la mejor desde el punto de vista del derecho constitucional a la constatación de que es debida iusfundamentalmente ( $\mathrm{ibid}$.). Si se presupone la posibilidad de un paso tal, es perfectamente posible partir en la argumentación jurídica del modelo de los valores en lugar del modelo de los principios». Y, en tercer lugar, porque «el hecho de que el concepto de principio, en menor medida que el de los valores, da lugar a menos falsas interpretaciones» (ibid.). A estas alturas de nuestro análisis, esta última ventaja resulta por lo menos dudosa, si se consideran los efectos que ha tenido la equivocidad del término «mandatos de optimización». Pero, ¿qué se puede decir en torno a las otras dos razones?

Por lo que respecta a la primera, la afirmación de que en la práctica jurídica de lo que se trata es de qué es «lo debido», debemos advertir que esto último puede entenderse en dos sentidos: como lo que debe ser o debe hacerse (ought to; sollen), o como lo consistente, de manera estricta, en deberes u obligaciones (duty; Pflicht), para emplear una potente distinción planteada por NINO (NINO, 2005: 37 y ss.). Si entendemos «lo debido» (en sentido denso) como lo consistente en deberes u obligaciones, habría que decir que en la práctica jurídica no se trata solamente de qué es «lo debido», por cuanto, como vimos hace poco, la corrección o proporcionalidad del juicio de precedencia condicionada y de la regla consiguiente (o sea, «lo debido») tiene como insumos justificativos valoraciones acerca del grado de intensidad de la intervención en los principios en colisión. Sin embargo, esta objeción podría ser esquivada si se asume «lo debido» (en sentido tenue) como lo que debe ser o debe hacerse. Esto, además, confirmaría la segunda ventaja señalada por ALEXY, la de la convertibilidad mutua entre principios y valores, de manera tal que un valor no sería sino el correlato necesario de un principio y viceversa (en el mismo sentido, vid. PECZENIK, cit.: 12).

Sin embargo, esto trae consigo una consecuencia importante para nuestra reconstrucción: la continuidad así trazada entre el plano axiológico y el deontológico hace que, en el modelo de la ponderación, los principios jurídicos no operen como enunciados estrictamente deónticos (deberes, prohibiciones, permisiones) pues, como se ha visto, su operatividad alude exclusivamente a su carácter de correlatos de valores. Antes mostramos, no obstante, que en los modelos de la adecuación, de la necesidad y de la colisión, los principios también operan como enunciados deónticos estrictos, además de como correlatos de valores: pueden operar como fines y como restricciones. Lo que revela que, en la teoría de los principios como mandatos de optimización, la operatividad de estos atañe tanto a «lo debido» como lo que debe ser o debe hacerse, cuanto a «lo debido» como lo consistente en deberes u obligaciones. En la faz deonto- 
lógica de los principios, entonces, habría dos niveles: un nivel deontológico tenue, en el que principios y valores son — por decir así- las dos caras de una misma moneda; y un nivel deontológico denso, en el que los principios adquieren la forma de estructuras deónticas prima facie que, si bien se fundamentan en valores, no son el mero correlato de estos. El postpositivismo de ALEXY, no obstante, no desarrolla su teorización en torno a esta escisión de niveles: ¿De qué naturaleza es la relación entre un nivel y otro? Metodológicamente, ¿cómo se transita de uno a otro? Según las particularidades del nivel deontológico denso, ¿qué clasificación de los principios cabría formular? ${ }^{16}$. Esta viene a ser nuestra observación final.

\section{CONCLUSIONES}

El postpositivismo de ALEXY tiene su núcleo en la tesis de que los principios son mandatos de optimización. La que los define según el rol operativo que, como razones para la acción, ellos cumplen según dos modelos argumentativos específicos: el modelo de la optimización fáctica y el modelo de la optimización jurídica o de la ponderación en sentido amplio. El primero comprende, a su vez, el modelo de la adecuación y el de la necesidad; y el segundo, el de la colisión y el de la ponderación en sentido estricto o balanceo. Los principios presentan dos tipos de operatividad, o como bienes o valores (en correspondencia con su nivel deontológico tenue), o como estructuras deónticas estrictas (en correspondencia con su nivel deontológico denso). En todos los modelos mencionados, la optimización exige (focalmente) que los principios operen como bienes o valores en cuya virtud se fundamentan juicios acerca del detrimento de los principios. En el caso de la optimización fáctica, los principios operan, además, como estructuras deónticas estrictas que consisten, ya en fines, ya en restricciones. Los indicados juicios acerca del detrimento de los principios permiten justificar: i) en la optimización fáctica, la corrección o proporcionalidad de excluir o no la norma o medida bajo examen por ser inadecuada o innecesaria para la realización de un determinado principio que opera como fin, dado que tal norma o medida socavaría otro principio que opera como restricción prima facie del anterior — modelos de la adecuación y de la necesidad—; ii) y en la optimización jurídica, ii.a) la afirmación de que la exclusión o no de esas normas o medidas, respectivamente, causarían detrimento, o bien, al principio que opera como fin, o bien, al que opera como restricción prima facie del primero - modelo de la colisión-, ii.b) la afirmación de que es correcto o proporcionado conceder, en el caso concreto, precedencia al primer principio respecto del segundo, o viceversa - modelo de la ponderación-, y ii.c) la afirmación de que es correcto, como producto deductivo de ii.a) y ii.b), excluir definitivamente o no la norma o medida bajo examen.

Sobre la teoría de los principios de ALEXY cabe hacer algunas observaciones críticas: ha usado el término «optimización» de manera equívoca, lo que ha oscurecido el concepto que dicho autor ha querido atribuirle. Esto ha llevado a entender la optimi-

16 Nótese que las clases de principios identificadas por AlEXY (formales, sustantivos, derechos individuales, bienes colectivos, etc.) aluden estrictamente al nivel deontológico tenue; ellas son, en el fondo, clases de bienes o valores. 
zación como maximización de un estado de cosas y, derivadamente, como fusión de estados de cosas a maximizar. Aunque nuestra reconstrucción indica que estas interpretaciones no serían acertadas, el modelo alexyano de la necesidad deja algún resquicio para pensar que su seguimiento puede conducir a una deriva maximizadora: la difuminación de los límites del conjunto de normas o medidas - por ejemplo- legislativas que el juez constitucional está autorizado a tener en cuenta al elaborar su juicio de necesidad. En cuanto al modelo de la colisión, ALEXY deja sin aclarar cuál es la índole de la relación entre la exclusión de la norma o medida bajo examen y el principio que opera como restricción prima facie; y, aunque es obvio que, por otro lado, la relación entre la norma o medida bajo examen y el principio que opera como fin es de carácter teleológico (medio-fin), ALEXY no aclara por qué, en su opinión, no es lo mismo el grado de realización de un principio que opera como fin y el grado de intervención en él provocado por otro que opera como su restricción prima facie; y tampoco explica cuáles serían las relaciones entre esos dos aspectos. Finalmente, ALEXY no teoriza suficientemente en torno a la separación entre los dos niveles deontológicos de los principios, el tenue y el denso; dejando sin desarrollar, por ejemplo, qué tipo de relación hay entre ellos, cuáles son los pormenores del flujo argumentativo que va del uno al otro, cómo se clasificarían los principios jurídicos a la luz de lo anterior, etcétera.

\section{REFERENCIAS BIBLIOGRÁFICAS}

Aguiló, J., 2015: El arte de la mediación: argumentación, negociación y mediación, Madrid: Trotta.

AleXY, R., 1988: «Sistema jurídico, principios jurídicos y razón práctica», en Doxa, 5, 139-151.

- 1993: «Derechos, razonamiento jurídico y discurso racional», en Derecho y razón práctica, México: Fontamara, 25-42.

- 2002a: «Epílogo a la teoría de los derechos fundamentales», en Revista Española de Derecho Constitucional, 22(1), 13-64.

- 2002b: Teoría de los derechos fundamentales, Madrid: Centro de Estudios Políticos y Constitucionales.

— 2003a: «Cinco preguntas a Robert Alexy (entrevista por Diana Quintero)», en Precedente Anuario Jurídico, 89-93.

- 2003b: «Derecho constitucional y derecho ordinario. Jurisdicción constitucional y jurisdicción ordinaria», en Tres escritos sobre los derechos fundamentales y la teoría de los principios, Bogotá: Universidad Externado de Colombia, 41-92.

— 2003c: «On Balancing and Subsumption. A Structural Comparison», en Ratio Juris, 16(4), 433-449.

- 2003d: «Sobre la estructura de los principios jurídicos», en Tres escritos sobre los derechos fundamentales y la teoría de los principios, Bogotá: Universidad Externado de Colombia, 93-139.

- 2005: «Ponderación, control de constitucionalidad y representación», en FONTAMARA (ed.), Teoría del discurso y derechos constitucionales, México, 89-103.

— 2007: «La fórmula del peso», en Teoría de la argumentación jurídica, 2. a ed., Madrid: Centro de Estudios Políticos y Constitucionales, 349-374.

- 2009: «Derechos fundamentales, ponderación y racionalidad», en Revista Iberoamericana de Derecho Procesal Constitucional, 11 (enero-junio), 3-14. 
— 2011a: «Algunas reflexiones en torno a cómo mi pensamiento jurídico ha ido desarrollándose con el paso de los años», en C. Bernal (ed.), La doble dimensión del Derecho. Autoridad y razón en la obra de Robert Alexy, Lima: Palestra, 50-88.

— 2011b: «Deber ideal», en L. Clérico, J.-R. Sieckmann y D. Oliver-Lalana (eds.), Derechos fundamentales, principios y argumentación jurídica. Ensayos sobre la teoría jurídica de Robert Alexy, Granada: Comares, 15-35.

- 2011c: «Los derechos fundamentales y el principio de proporcionalidad», en Revista Española de Derecho Constitucional, 91 (enero-abril), 11-29.

- 2011d: «Sobre la estructura de los derechos fundamentales de protección», en J.-R. SIECKMANN (ed.), La teoría principialista de los derechos fundamentales, Madrid: Marcial Pons, 119-136.

— 2014: «Sobre o conceito de principio jurídico», en A. Travessoni y G. Trivisonno (eds.), Teoria Discursiva do Direito, Rio de Janeiro: Editora Forense, 203-242.

AquinO, T. DE, 1997: «In V Ethicorum (Sententia Libri Ethicorum)». Recuperado febrero 1, 2016, de bttp://www.documentacatholicaomnia.eu/03d/1225-1274,_Thomas_Aquinas,_Aristotelis_Libri._Sententia_Libri_Ethicorum,_LT.pdf.

— 1998: «De Regimine Principum Ad Regem Cypri». Recuperado febrero 1, 2016, de bttp:// www.documentacatholicaomnia.eu/03d/1225-1274,_Thomas_Aquinas,_De_Regimine_Principum._ad_Regem_Cypri,_LT.pdf.

ARISTÓTELES, 1993: Ética Nicomáquea. Madrid: Gredos.

AtienZA, M., 2006: El Derecho como argumentación. Barcelona: Ariel.

— 2007: «Argumentación y constitución», en Fragmentos para una teoría de la constitución, Madrid: Iustel, 113-181.

- 2010: «A vueltas con la ponderación», Anales de la Cátedra Francisco Suárez, 44, 43-59.

- 2013: Curso de Argumentación Jurídica, Madrid: Trotta.

- 2014a: «Ni positivismo jurídico ni neoconstitucionalismo: una defensa del constitucionalismo postpositivista», en Observatório Da Jurisdição Constitucional, 7(2), 1-24.

— 2014b: «Ponderación y sentido común jurídico». Recuperado febrero 1, 2016, de bttp:// dfddip.ua.es/es/documentos/ponderacion-y-sentido-comun.pdf?noCache $=1415615082659$.

Atienza, M., y Ruiz Manero, J., 2004: Las piezas del Derecho. Teoría de los enunciados jurídicos,

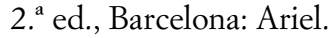

- 2009: «A modo de epílogo. Una conversación sobre el Derecho y otras varias cosas», en M. Atienza y J. Ruiz Manero (eds.), Para una teoría postpositivista del Derecho, Lima: Palestra-Temis, 259-302.

BAYÓN, J. C., 1991: La normatividad del Derecho: deber jurídico y razones para la acción, Madrid: Centro de Estudios Constitucionales.

BERNAL, C., 2007: El principio de proporcionalidad y los derechos fundamentales, 3. ${ }^{a}$ ed., Madrid: Centro de Estudios Políticos y Constitucionales.

CiCERÓn., 1877: De officiis, New York: D. Appleton and Company.

DwORKIN, R., 1967: «The Model of Rules», en University of Chicago Law Review, 35(1), 14-46.

FERRAJOLI, L., 2011: «Constitucionalismo principialista y constitucionalismo garantista», en Doxa, 34, 15-53.

García Figueroa, A., 1998: Principios y positivismo jurídico, Madrid: Centro de Estudios Políticos y Constitucionales.

Habermas, J., 2005: Facticidad y validez. Sobre el derecho y el Estado democrático de derecho en términos de teoría del discurso, Madrid: Trotta.

LOPERA, G., 2004: «Los derechos fundamentales como mandatos de optimización», en Doxa, 27, 211-243. 
LOZADA, A., 2012: «Sobre la dimensión argumentativa del Derecho», en J. MonTAÑA (ed.), Apuntes de derecho procesal constitucional, t. I, Quito: Centro de Estudios y Difusión del Derecho Constitucional.

- 2016: Derechos y constitucionalismo discursivo, Universidad de Alicante. Recuperado de bttps://rua.ua.es/dspace/bitstream/10045/54935/1/tesis_lozada_prado.pdf.

Maquiavelo, N., 2011: «El príncipe», en Maquiavelo, Madrid: Gredos.

NinO, C. S., 2005: Ética y derechos bumanos. Un ensayo de fundamentación, 2." ed., Buenos Aires: Astrea.

Nozick, R., 1974: Anarchy, State, and Utopia, Oxford: Blackwell.

PeczeniK, A., 1992: «Los principios según Atienza y Ruiz Manero», en Doxa, 12, 327-331.

Platón, 1981: «Protágoras», en Diálogos I, Madrid: Gredos.

Poscher, R., 2011: «Aciertos, errores y falso autoconcepto de la teoría de los principios», en J.-

R. SIECKMANN (ed.), La teoría principialista de los derechos fundamentales, Madrid: Marcial Pons, 71-92.

PRIETO, L., 1992: Sobre principios y normas. Problemas del razonamiento jurídico, Madrid: Centro de Estudios Constitucionales.

RAZ, J., 1991: Razón práctica y normas, Madrid: Centro de Estudios Constitucionales.

RUIZ MANERO, J., 1990: Jurisdicción y normas: dos estudios sobre función jurisdiccional y teoría del derecho, Madrid: Centro de Estudios Constitucionales.

SieCKMANN, J.-R., 2006: «Sobre la fundamentación de los juicios ponderativos», en C. CALDERÓN (ed.), El modelo de los principios, Bogotá: Universidad Externado de Colombia, 159-204.

TARSKI, A., 1977: Introducción a la Lógica, Madrid: Espasa-Calpe. 